the frequency of the atom and the number of free electrons are not the same functions of the magnetic field at different temperatures and tensions this form of explanation might give a reason for the variation of the thermoelectromotive force and for the influence of tension on it.

Ohio State University.

\title{
Positive Ray Analysis of Lithium and Zinc.
}

By A. J. Dempster.

Positrvely charged atoms are formed by vaporizing the metal in an electrically heated capsule, and bombarding the vapor with electrons from a hot cathode. After falling through a definite potential the particles are deflected through $180^{\circ}$ by a magnetic field, the semicircular path being smaller for the lighter than for the heavier. The different atoms are observed by means of their charge. Two components were observed in the case of lithium with atomic weights 6 and 7 . The proportion of the two components was found to vary in different experiments. Preliminary experiments with zinc have given three strong components two units apart in atomic weight, and one weaker component one on the heavy side of the strong group and separated also by two units of atomic weight.

RYERSON LABORATORY,

University OF Chicago.

\section{A Convenient Contactor for Small Currents.}

By Frederick J. Schlink.

ORDINARY drawing ink applied in the form of lines with a ruling pen will form an electrical path of sufficient conductivity to be used in connection with the oscillograph for recording the instants at which the lines pass by a brush or wiper. As it is frequently necessary to determine electrically the rate of translation or rotation of some moving part, the possibility of providing for the electrical contacts required by simply drawing lines on a strip of paper gummed to the moving part, affords great convenience, in comparison with the trouble and expense of constructing the usual composite commutator for the purpose. Moreover, the ink and paper contacting device will of ten be of negligible mass and inertia in relation to the part whose motion is under investigation. The lines may be connected to a common return circuit by drawing transversely across them a wide band of the same material, which in turn may be connected to a metallic return path through wire, metallic foil, or similar connection.

The contact pressures required will be extremely minute, and as the ink and paper are sensibly in the same plane, difficulty from bouncing of the brush will not be encountered unless the velocities are very high indeed. It is found that the drawing ink will carry a considerable amount of graphite in suspension; addition of this will increase the conductivity markedly, especially if the ink after drying be burnished and compacted to a glossy condition by the use of a smooth metallic tool. 